\title{
An autopsy case of metastatic extramammary Paget's disease treated with multimodality treatment including anti-HER2 therapy: What is the clinical and pathological significance of trastuzumab to the patient?
}

\author{
Noriko Yoshimura ${ }^{1^{*}}$, Koji Arihiro $^{2}$, Shunsuke Takahagi ${ }^{3}$, Michihiro Hide $^{3}$ \\ ${ }^{1}$ Department of Surgery, Hiroshima General Hospital, Hiroshima, Japan; *Corresponding Author: yoshimuranoriko32@gmail.com \\ ${ }^{2}$ Department of Pathology, Hiroshima University Hospital, Hiroshima, Japan \\ ${ }^{3}$ Department of Dermatology, Hiroshima University Hospital, Hiroshima, Japan
}

Received 1 October 2013; revised 21 October 2013; accepted 27 October 2013

Copyright (C) 2013 Noriko Yoshimura et al. This is an open access article distributed under the Creative Commons Attribution License, which permits unrestricted use, distribution, and reproduction in any medium, provided the original work is properly cited.

\begin{abstract}
Advanced Extramammary Paget's disease (AEMPD) shows a poor prognosis despite multimodality therapy. In recent years, it is suggested that anti-HER2 therapy may be promising for HER2-positive AEMPD. We herein present an autopsy case of a patient with AEMPD treated with multimodality treatment including anti-HER2 therapy. A 78-year-old man who diagnosed with AEMPD died after surgery and systemic chemotherapy including anti-HER2 therapy (trastuzumab). The metastatic skin lesions were immnohistologically HER2-positive. While the patients were administrated trastuzumab plus taxan (docetaxel, and paclitaxel) regimen, the metastatic skin lesion decreased, however, brain metastases were found in his brain and trastuzumab is discontinued. The skin metastasis rapidly spread over his body, leading to weakness, and he eventually died. At autopsy, the lesions of EMPD were extended distant organs including brain, although each metastasis was small and asymptomatic. The wide lesion of skin metastasis was exacerbated after discontinuation of trastuzumab, and transudate was observed due to the extensive necrosis and erosion. Our autopsy findings showed one progressive pattern of AEMPD, and indicated what is the clinical and pathological significance of anti HER2 therapy for HER2-positive AEMPD.
\end{abstract}

Keywords: Extramammary Paget's Disease; HER2; Autopsy

\section{INTRODUCTION}

Extramammary Paget's disease (EMPD) is a distinct type of skin carcinoma originating in the apocrine gland. Advanced EMPD (AEMPD) cases show a poor prognosis despite multimodality therapy. In recent years, some authors reported the overexpression of human epidermal growth factor receptor 2 (HER2) [1-3] in AEMPD, thus suggesting that anti-HER2 therapy may be promising for the disease. We herein present an autopsy case of a patient with AEMPD who was treated with multimodality therapy, including trastuzumab. The detailed clinical presentation of this patient has been reported previously [4]. In this letter, we highlight the autopsy findings of our patient and examine the clinical and pathological significance of trastuzumab to the patient.

\section{CASE}

A 78-year-old male patient presented with erythema on the scrotum, which he had first noted almost five years prior. The erythema had gradually extended, these lesions were diagnosed as EMPD by a skin biopsy 28 months before his death and skin resection and dissection of the left inguinal lymph nodes were performed. After surgery, the patient was treated with systemic chemotherapy using Mitomycin $\left(20 \mathrm{mg} / \mathrm{m}^{2}\right)+5-\mathrm{FU}\left(1000 \mathrm{mg} / \mathrm{m}^{2}\right)$ at first, and then weekly with Docetaxel $\left(60 \mathrm{mg} / \mathrm{m}^{2}\right)$. However, the skin recurrence worsened. At this point, a 
biopsy of these skin lesions was performed, and it revealed HER2 overexpression on the tumorcellular membrane. The patient was therefore administrated an anti-HER2 drug, trastuzumab ( $4 \mathrm{~kg} / \mathrm{kg}$ ), followed by a 2 $\mathrm{mg} / \mathrm{kg}$ maintenance dose at weekly intervals in combination with Taxanes (Docetaxel and Paclitaxel) according to a protocol for HER2-positive metastatic breast cancer beginning 14 months prior to his death. The metastatic skin lesion rapidly decreased in size. However, multiple small spots were found in his brain by MRI 10 months before his death, which were diagnosed as brain metastases. The regimen was then shifted to low dose 5-FU (1000 mg/m $\mathrm{m}^{2}$ ) and Cisplatin $\left(15 \mathrm{mg} / \mathrm{m}^{2}\right)$. Radiotherapy for the brain was performed. The skin recurrence rapidly spread over his body, and he suffered from gradual debilitation, pneumonia, a urinary tract infection, and eventually died.

We performed an autopsy on the patient. For the skin lesion, HER2-positive skin recurrence was observed extending to the vulva, bilateral thighs and the lower abdomen (Figure 1). These exacerbated after discontinuation of trastuzumab, and transudate was observed due to the extensive necrosis and erosion. For brain metastases, some small lesions were detected in the basal ganglion, hippocampus, cerebellum, and pons. Some of them exhibited vacuolar degeneration, indicating the effect of whole brain radiotherapy (Figure 2). In the lungs, acute bronchopneumonia and acute diffuse alveolar damage with pleural effusion were detected. In the kidneys, acute pyelonephritis and mild acute tubular necrosis were observed. Other metastases were found in bilateral lungs, the pancreas, and lymph nodes.

\section{DISCUSSION}

Our patient died 28 months after the diagnosis of AEMPD. To the best of our knowledge, there are no reports about autopsy case of AEMPD treated with antiHER2 therapy. Generally, most AEMPD cases are difficult to treat even with multimodality therapy. The mortality rate associated with progressive EMPD is 13\% -

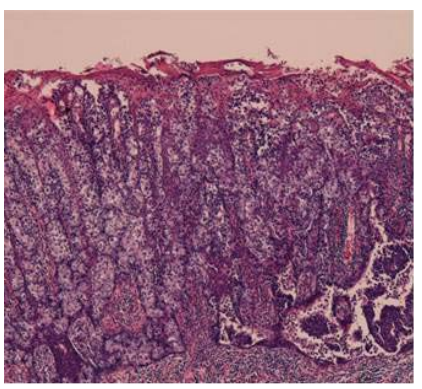

(a)

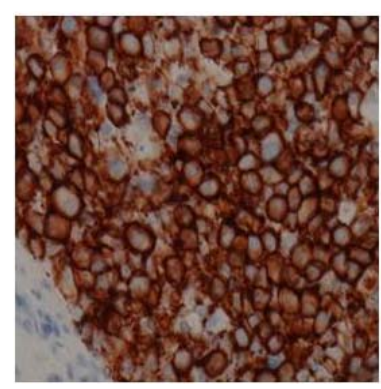

(b)
Figure 1. Histopathologic findings: (a) H.E stain $\times 40$, (b) Immunohistochemical stain of skin lesion revealed HER2 were positive.

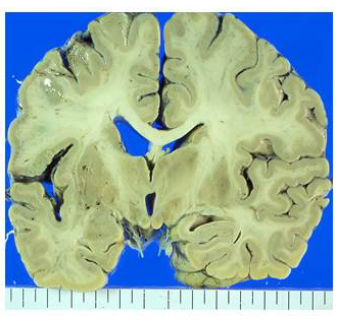

(a)

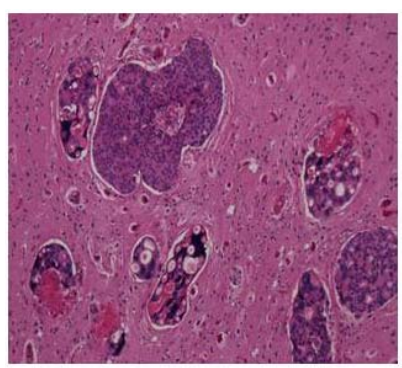

(c)

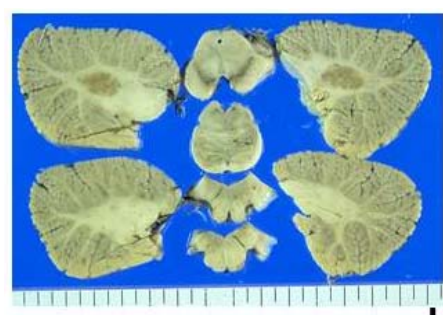

(b)

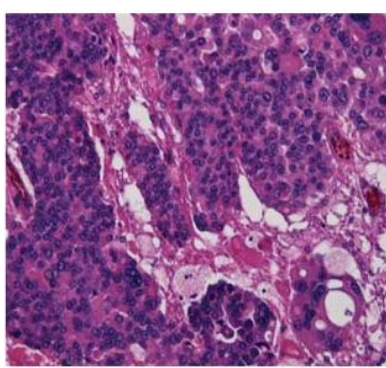

(d)
Figure 2. Macroscopic findings: (a) Cerebrum; (b) Cerebellum. small, multipulmetastatic nodules were found. Histopathological findings; (c) Basal ganglion (H.E stain $\times 40$ ); (d) hippocampus $($ H.E stain $\times 100)$. The vacuolar degenerations were found in some metastasis.

$18 \%[5,6]$, and the 5-year survival rate of AEMPD is reported to be $72 \%$ [7]. The general pattern of metastasis in EMPD is lymphogenous, and less often, hematogenous spread. Brain metastasis is relatively uncommon. Furthermore, the detection of brain metastasis during the course of disease is unlikely. There is no established chemotherapy regimen for AEMPD and various regimens have been administered, such as topical 5-Fluorouracil, Mitomycin C, Cisplatin, Docetacel, etc. As noted previously, 20\% - 60\% of EMPD show HER2 expression, which is approximately the same or higher percentage than primary breast cancers. The combination of conventional chemotherapy regimens with anti-HER2 drugs is therefore expected to improve the overall survival for AEMPD patients. Previous reports show favorable local control, but further investigations, such as those evaluating the response rate or survival, are difficult due to the relative rarity of the condition. In our patient, the use of trastuzumab plus taxanes showed a dramatic effect on the skin metastasis, but brain metastases developed. Is this situation coincidental? Trastuzumab does not cross the blood-brain barrier and it has been reported that patients with HER2-positive breast cancer have a significantly higher incidence of brain metastasis after treatment with trastuzumab [8]. Nevertheless, it has been suggested that the metastasis of EMPD to the brain is rare. However, since each metastasis was small and did not lead to neurological symptoms, we may not have had to discontinue administering trastuzumab. It might have been the transudate from the skin metastasis that caused low nutrient 
condition, generalized weakness, and eventually death.

In many AEMPD cases, there have been no reports on lethal events such as hemorrhage due to metastases to other organs that resulted in death. Rather, patients progressively weaken and die. Hence, we suggest that control of skin lesions be assigned a priority instead of focusing on treating small metastases in other organs.

New anti-HER2 drugs have been developed recently. Lapatinib, for example, is a small molecule anti-HER2 agent used for breast cancer which is known to penetrate the blood-brain barrier and contribute to overall survival of patients with brain metastases. Although clinical indications need to be clearer and the cost might hinder positive use, lapatinib could be an effective treatment strategy for AEMPD.

\section{CONCLUSION}

We confirmed one progressive pattern of AEMPD treated with multimodality therapy, including anti-HER2 therapy. More amassed reports and further investigations will be necessary.

\section{REFERENCES}

[1] Ogawa, T., Nagashima, Y., Wada, H., Akimoto, K., Chiba, Y. and Nagatani, T. (2005) Extramammary Paget's: Analysis of growth signal pathway from the human epidermal growth factor receptor 2 protein. Human Pathology, 36, 1237-1280.

[2] Plaza, J.A., Torres-Cabala, C., Ivan, D. and Prieto, V.G.
(2009) HER-2/neu expression in extramammary Paget disease: A clinicopathologic and immunohistochemistry study of 47 cases with and without underlying malignnancy. Journal of Cutaneous Pathology, 36, 729-733. http://dx.doi.org/10.1111/j.1600-0560.2008.01148.x

[3] Richter, C.E., Hui, P., Buza, N., Silasi, D.A., Azodi, M., Santin, A.D., et al. (2010) HER-2/NEU overexpression in vulvar Paget disease: The Yale experience. Journal of Clinical Pathology, 63, 544-547. http://dx.doi.org/10.1136/jcp.2010.077446

[4] Takahagi, S., Noda, H., Kamegashira, A., Madokoro, N., Hori, I. and Shindo, H. (2009) Metastatic extramammary Paget's disease treated with paclitaxel and trastuzumab combination. The Journal of Dermatology, 36, 457-461. http://dx.doi.org/10.1111/j.1346-8138.2009.00676.x

[5] Chanda, J.J. (1985) Extramammary Paget's disease: Prognosis and relationship of internal malignancy. Journal of the American Academy of Dermatology, 13, 1009-1014. http://dx.doi.org/10.1016/S0190-9622(85)70254-X

[6] Hatta, N., Yamada, M., Hisano, T., Fujimoto, A. and Morita, R. (2008) Extramammary Paget's disease: Treatment, prognosis and outcome in 76 patients. British Journal of Dermatology, 158, 313-318.

[7] Siesling, S., Elferink, M.A.G., van Dijck, J.A., Pierie, J.P. and Blokx, W.A. (2007) Epidemiology and treatment of extramammary Paget disease in the Netherlands. European Journal of Surgical Oncology, 33, 951-955. http://dx.doi.org/10.1016/j.ejso.2006.11.028

[8] Musolino, A., Cinnolallo, L., Panebianco, M., Fontana, E., Zanoni, D., Bozzetti, C., et al. (2011) Multifactorial central nervous system recurrence susceptibility in patients with HER2-positive breast cancer. Cancer, 117, 18371846. http://dx.doi.org/10.1002/cncr.25771 